\title{
Orbital prosthesis fabrication: current challenges and future aspects
}

This article was published in the following Dove Press journal:

Open Access Surgery

21 April 2016

Number of times this article has been viewed

\section{Binit Shrestha \\ Sita Thaworanunta}

Maxillofacial Prosthetic Service, Department of Prosthodontics, Faculty of Dentistry, Mahidol University, Bangkok, Thailand
Correspondence: Sita Thaworanunta Maxillofacial Prosthetic Service, Department of Prosthodontics, Faculty of Dentistry, Mahidol University, Sixth Yothi Street, Rachathewi, Phayathai, Bangkok 10400, Thailand $\mathrm{Tel}+66818480608$

Email thitypa@hotmail.com

\begin{abstract}
Orbital defect, arising from tumor-related resections, trauma, and congenital anomalies, can lead to negative functional and psychological effects in the patients. Rehabilitation with an orbital prosthesis can provide satisfactory aesthetic outlook and can be combined with surgical reconstruction to improve the retention. Despite less than ideal properties, silicone elastomer is the most commonly used material to fabricate orbital prosthesis. Several studies have been conducted on developing newer materials and improving the properties of silicone elastomer. Craniofacial implants offer greater retention compared to adhesives, but they have been observed to have a higher risk of implant failure and related side effects when placed in irradiated bone. This review gives a scope on understanding the current challenges faced during fabrication of orbital prosthesis and the future directions of this field.
\end{abstract}

Keywords: orbital defect, silicone prosthesis, CAD/CAM, craniofacial implants

\section{Introduction}

Orbital defects mostly arise from management of tumors originating from the orbital contents, or due to the spread of tumors originating from paranasal sinus, palate, nasal cavity, overlying skin, and intraoral mucosa. They can also result from midfacial trauma associated with orbital fractures and damage to the orbital contents, and related surgeries. These defects lead to significant facial disfigurement, functional limitations, and negative psychological impact on the patient. Reconstruction is carried out 1) to obtain a clear separation between the oral and nasal cavities to allow unobstructed and unimpaired breathing and 2) to obtain acceptable aesthetics. Various types of flaps, such as anterolateral thigh flaps, radial forearm flap, and fibular flap, have been utilized for the reconstruction of midface and orbit. ${ }^{1,2}$ Eyelid reconstruction has also been attempted to retain an ocular prosthesis. ${ }^{3,4}$ However, due to the complexity, most of the procedures are limited to coverage of the defect and exposed structures with microvascular free flaps.

Prosthetic rehabilitation is an alterative treatment option to surgical reconstruction. These prostheses mimic the patient's missing structures and have acceptable aesthetic outlook. They also permit hygiene maintenance around the defect and observation for tumor recurrence. A close cooperation and communication between the maxillofacial surgeon and maxillofacial prosthodontist is required so that retentive foundations can be made during the time of initial surgery. They include creating undercuts behind the supra- or infra-orbital rim, covering exposed bone surfaces with skin grafts, and/or building up adequate viable bone to receive endosseous implants. ${ }^{5}$ As compared to the conventional treatment, the use of digital imaging and rapid prototyping techniques has 
improved treatment predictability and accuracy, as the entire surgical procedures can be preplanned such that the required optimal retention and aesthetic outlook of the prosthesis are achieved. However, there are also drawbacks to this treatment. This literature review deals with the current challenges faced during the fabrication of an orbital prosthesis and its future aspects.

\section{Maxillofacial prosthetic materials}

An ideal maxillofacial prosthesis material should have ideal mechanical and physical properties, such as dimensionally stable, high edge tear strength, high resistance to abrasion, high tear and tensile strength, low coefficient of friction, low specific gravity, low surface tension, low thermal conductivity, no water sorption, odorless, nonflammable, life-like translucency, softness comparable to skin, and variable flexibility. It should also be biocompatible with the surrounding tissues without release of any allergenic or toxic by-products after curing. It should allow cleansing without loss of surface texture or margins and should be color stable and resistant to environmental discoloration and microbial growth. ${ }^{5}$

Methyl methacrylate, polyurethanes, and silicone elastomer have gained the most popularity as maxillofacial prosthetic materials ${ }^{6}$ as they can be intrinsically and extrinsically tinted using oil-based or dry earth pigments to match the patient's skin. Methyl methacrylate is readily available, durable, and compatible with the tissues, but it is hard, lacks texture, and is difficult to match color. Duplication is also not possible as the processing mold is normally damaged during processing. Polyurethanes have high tear strength, superior esthetics, good ultimate strength, and elongation, but they are moisture sensitive during curing, resulting in bubbles and loss of mechanical properties. ${ }^{7}$ Silicone elastomer may not be as durable as methyl methacrylate, but it is easy to color and has better cosmetic outlook, as the margins of prosthesis can be made thin and translucent to blend with the surrounding skin. The major drawbacks of silicone elastomers are their low tear and edge strengths and relatively low elongation, which make them highly susceptible to tearing, especially the margins of an adhesive-retained prosthesis during daily placement and removal. Silicone can be further grouped into heat temperature vulcanizing and room temperature vulcanizing (RTV). According to a survey by Montgomery and Kiat-Amnuay, ${ }^{8}$ MDX4-4210 and A-2186 RTV silicones along with Silastic Medical Adhesive Type A were the most preferred maxillofacial prosthetic materials. Six months to 1 year following use, silicone prostheses start to lose their color stability and degenerate, requiring additional extrinsic staining or even refabricating. ${ }^{9}$
Various new materials have been developed and tested for their use in this field. ${ }^{10,11}$ Similarly, modifications have been attempted on existing materials to improve their physical properties. Udagama developed a technique to bond thin urethane liner to silicone prosthesis, which enhanced the edge strength and tear resistance. The technique improved the surface's wettability, which enhanced the bonding with adhesives, ${ }^{12}$ but weak bond between urethane and silicone led to frequent separations. Ultraviolet (UV) light absorbing agents and opacifiers have been added to silicone to slow the color disintegration but the results have been variable; some studies showed protective effects of these agents against UV-induced discoloration, ${ }^{13-15}$ whereas some showed no beneficial effects. ${ }^{16,17}$ Recent modifications include addition of nanoparticles to the elastomer composition. Han et al incorporated nano-oxides of $\mathrm{Ti}, \mathrm{Zn}$, or $\mathrm{Ce}$ at concentrations of $2.0 \%$ and $2.5 \%$ by weight to silicone A-2186 maxillofacial elastomer and noted improved tensile and tear strengths and percentage of elongation. Conversely, the addition of nano-oxides increased the hardness of the material. ${ }^{18}$ Despite many studies, to date, none of the commercially available materials satisfy all the requirements of the ideal maxillofacial material.

\section{Adhesive-retained prosthesis}

The fabrication of an orbital prosthesis first begins by obtaining an impression of the orbit and periorbital area with least distortion of the surrounding tissues. The conventional method utilizes different impression materials and techniques to obtain the impression. Type II dental stone (eg, impression plaster) supported by a thin layer of gauze strips has been traditionally used to obtain a facial moulage. Irreversible hydrocolloid (eg, alginate) can serve as a cost-effective alternative impression material, ${ }^{19}$ but the material needs to be supported by a customized tray to prevent overflow. It is also susceptible to distortion if not poured immediately. Polysulfide impression material supported by a layer of fast setting impression plaster and light- and heavy-bodied polyvinyl siloxane impression material have also been used. Digital scanning techniques have overcome many of the limitations of these impression materials and are discussed in more detail in the later sections of this article.

With the conventional techniques as outlined in Figure 1, the obtained impression needs to be poured with dental stone to obtain a working cast. A stock or customized ocular prosthesis that represents the patient's normal eye is obtained and oriented in wax sculpture. It is then hand carved to reproduce all of the patient's anatomy, such as eyelids, skin folds, and 


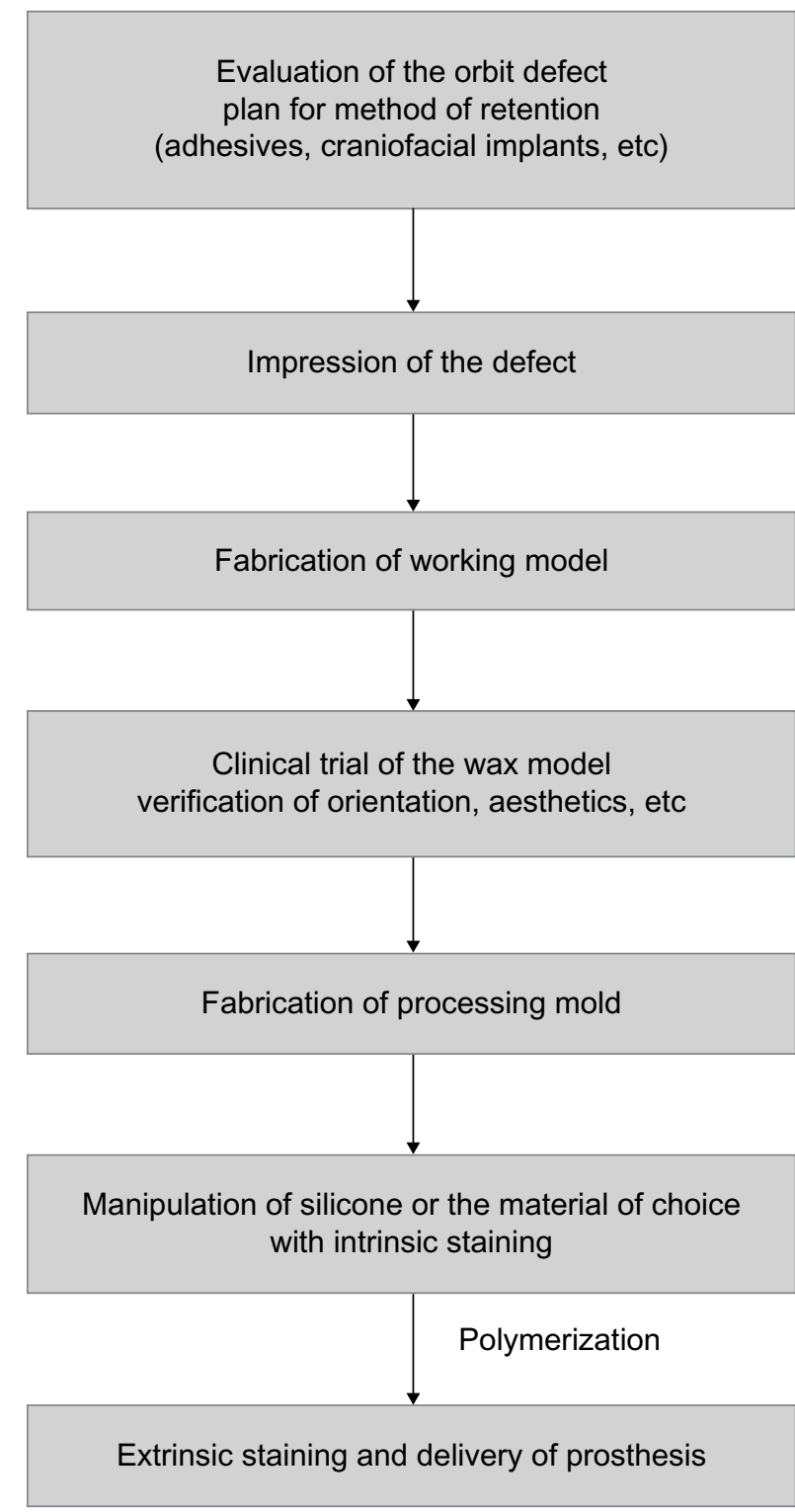

Figure I Outline for the fabrication of an orbital prosthesis using conventional technique.

texture, and clinically verified for overall aesthetics and adaptation. The sculpturing process is very subjective and depends on the skills of the operator. The wax sculpture is invested in dental stone to fabricate a mold. Silicone elastomer or the material of choice is intrinsically colored to match the shade of the patient and packed. Intrinsic staining is important, as it is more resistant to the effects of environment and handling. UV absorbing agents and opacifiers can be added to the silicone elastomer at this stage. Type and concentration of pigments can also influence the elastic and viscous portions of silicone as noted by $\mathrm{Hu}$ et al. ${ }^{20}$ Following polymerization, the prosthesis is extrinsically stained with a sealer, such as Silastic Medical adhesive Type A (Dow Corning, Auburn, MI, USA) RTV silicone. ${ }^{8}$ Artificial eyelids and eyelashes can be further added to give a natural appearance to the orbital prosthesis.

Various types of medical grade adhesive systems are commercially available, such as spray-on adhesives, pastes, double-sided adhesive tapes, and liquid emulsions to retain the prosthesis. The main drawback of these adhesive systems is that they become less effective when the margins of the prosthesis extend beyond the orbit into movable tissue bed areas. Loss of adhesiveness can lead to open junctions and eventual dislodgement of the prosthesis.

\section{Implant-retained prosthesis}

Craniofacial implants are made of titanium alloys, which osseointegrate with the bone. The conventional craniofacial implants are $3-5 \mathrm{~mm}$ long with threaded and machined surface (Branemark System; Noble Biocare, Zürich-Flughafen, Switzerland). A flanged design was also used as a fail-safe feature to prevent accidental penetration of the implant into deeper structures, such as the brain. ${ }^{21}$ The newer craniofacial implants (EO System; Straumann, Basel, Switzerland) have sandblasted, large-grit, and acid-itched surface and allow for a faster osseointegration as compared to the machined surface. The use of craniofacial implants for retention of orbital prosthesis frees the patient from the use of adhesives or dependency on spectacle frameworks. Mechanical and chemical irritation associated with the use of adhesives can also be avoided. Moreover, the aesthetic outcome is also improved, as the margins of the prosthesis can be thin, translucent, and blended with the surrounding skin. A quality-of-life study by Chang et a ${ }^{22}$ showed a better treatment satisfaction with implant-retained prosthesis as compared to conventional adhesive-retained prosthesis.

Due to the limited amount and curvature of the orbital bone, the placement of implants requires a multidisciplinary team approach toward treatment planning and should also take into account patient-, defect-, and tumor-related factors. Clinical evaluation along with radiographic investigation, such as computer tomography (CT) scan, should be performed to confirm the bone volume and density. Orbital rim has dense cortical bone but with limited volume. Generally three to four implants placed along the supraorbital and lateral aspect of the infraorbital rims are considered adequate for the retention of an orbital prosthesis. If the bone is adequate, longer implants can also be placed ${ }^{23}$ to gain maximum possible bicortical anchorage. During surgical placement, the angulation should also be adjusted to face the internal surfaces of the cavity so that the aesthetics of the future prosthesis are not compromised. 


\section{Placement of implants in irradiated bone}

Most of the tumor-related orbital defects also receive radiation therapy as a part of the treatment. Ionizing radiation leads to reduction in the number of viable osteoblasts and osteocytes and development of areas of fatty degeneration within the marrow spaces. In addition, blood vessels undergo progressive endarteritis, hyalinization, and fibrosis; thus, resulting in regional ischemia ${ }^{24}$ and a diminished osseous remodeling capacity. ${ }^{25}$ Hyperbaric oxygen (HBO) therapy is considered to counteract some of the negative effects of radiation therapy by improving angiogenesis and stimulating bone growth and turnover, ${ }^{26}$ but the protocol regarding its use in the placement of implant in irradiated bone has not been fully established.

Nishimura et al conducted a study where 23 implants were placed in irradiated (45-60 Gy) and nonirradiated orbital defects. The overall survival rate of the implants was 33.3\% for irradiated bone and $37.5 \%$ for nonirradiated bone with a follow-up period of 7 years. ${ }^{25}$ Similarly, in a study by Moran et al, 25 implants were placed in six patients with orbital defect. Of these patients, five had a history of postoperative radiation therapy (45-66 Gy). Although HBO therapy was not used, survival rates of $90 \%$ for irradiated bone and $100 \%$ for nonirradiated bone were noted at the end of the average follow-up period of 44.2 months. ${ }^{27}$ Curi et al conducted a study in which intraoral dental implants were placed in the orbital region with a one-staged surgery protocol. HBO therapy was used as a standard protocol for the irradiated patients. A high survival rate was observed in both irradiated and nonirradiated patients over a 2 -year recall period, with only one implant failure after 6 months of functional loading and one incidence of osteoradionecrosis (ORN) 6 months after prosthesis wear. ${ }^{23}$

A retrospective multicenter surgery was carried out in the United States on the use of craniofacial implants for the prosthetic rehabilitation of orbital defects; among the ten centers that took part in the study, an overall $73.2 \%$ success rate was noted over a mean follow-up period of 52.6 months. In this study, 92 implants were placed in irradiated bone (39.6-80.5 Gy), out of which only 21 implants were placed under HBO therapy, whereas 70 implants were placed without HBO therapy. Although previous reports have suggested that there is an increased risk of implant failure and ORN beyond $50 \mathrm{~Gy}$ of radiation, ${ }^{21}$ the study observed no significant relationship between the use of HBO therapy, implant location, radiation therapy history, and survival outcomes of implants placed in orbital bone. ${ }^{28}$
Overall, the success rates of implants in the orbital bone reported in the literature are between $33.3 \%$ and $96.4 \%$ in the irradiated bone and between $37.5 \%$ and $100 \%$ in the nonirradiated bone. ${ }^{25,27,29}$ On the contrary, a steady loss of implants in the orbital region has also been noted over long follow-up periods as compared to the auricular and nasal regions. ${ }^{25,30}$ Long-term studies are imperative to fully understand the effects of irradiation on craniofacial osseointegration. As a precaution, placing of implants in irradiated patients should be carried in institutions that are capable of handling these patients, as evidence points to a greater risk of implant failure and other radiation therapyassociated complication, such as ORN. Besides bone quality and quantity, treatment planning should also take into account patient-related factors such as patient health, motivation, age, and systemic diseases to judge whether the patient truly benefits from the treatment.

\section{One-staged and two-staged implant placement}

The implant placement in the orbital region is generally carried out under a two-staged protocol. The first step consists of implant placement and closure of the osteotomy site. After 4-6 months of healing, the implants are exposed, and transcutaneous healing abutments are attached to the fixture. Reduction in the periabutment tissues to $2-3 \mathrm{~mm}$ thickness or placement of a split-thickness skin graft helps to create thin immobile soft tissues, which, in turn, creates a better seal at the implant abutment interface and also minimizes lateral loading on the implants. The prosthetic rehabilitation with silicone prosthesis can then be initiated following the healing of the soft tissues. ${ }^{31}$ A single-staged protocol has also been utilized, where the implant placement, dermal reduction, and attachment of the abutments were carried out in a single setting. ${ }^{32}$

\section{Comparison of retentive elements}

An implant-retained facial prosthesis can be held by using either bar and clip or magnetic attachments. During placement of multiple craniofacial implants, it may be very difficult to obtain parallelism between them due to the curvature of the orbital bone. Bar and clip attachment splints the implants together using a metal framework, which helps to distribute the load among the implants. It is useful in restoration of large facial defects or when the retentive requirement is high. However, the bar design is becoming increasingly obsolete because it is technically sensitive to fabricate and misfit of the bar framework can jeopardize osseointegration. 
Hygiene maintenance is also difficult underneath a bar and clip attachments, as most of the patients who receive these prostheses are old with limited manual dexterity. Furthermore, monocular vision may also hamper their ability to clean. ${ }^{5}$

In most situations, the use of magnetic attachment is considered adequate for the retention of an orbital prosthesis. The implants are placed in both superior and inferior orbital rims to provide tripodal stabilizing effects. Magnets also exert a low moment force on the abutments and have a low profile that can be suitable for shallow defects with insufficient space for a bar construction. Generally, magnets follow a nonsplinted design, which makes it easier to maintain the hygiene as compared to a bar and clip attachment. Since the magnetic attachments are self-aligning, it is ideal for patients with limited dexterity.

For multiple implants, the angulation of the magnets at the orbital region may not be in an ideal plane. They may be slightly inward or outward depending on the available bone of the osteotomy site. A protrusive angulation of an implant can interfere with contour and esthetic requirement compromising an ideal shape of the prosthesis. In these situations, console abutment (Nobel Biocare, AB, Göteborg, Sweden) with offset extensions $\left(30^{\circ}, 60^{\circ}, 90^{\circ}\right.$, and $\left.110^{\circ}\right)$ for retentive components (eg, Magna Cap; Technovent, South Wales, UK) can be utilized. ${ }^{36}$ This allows for adjustments of the retentive elements to obtain a uniform path of placement and removal - one that offers the maximum amount of retention. On the other hand, console abutments can cause uneven distribution of load along the fixture, which can lead to cantilevering effects and long-term negative effects to the survival of the implant. ${ }^{26}$

The retentive elements corresponding to the bar and clip or magnets are enclosed in a polymethyl methacylate housing, which is then chemically bonded with the help of a primer to the silicone elastomer. Primer is silane-coupling agent with organic and inorganic parts and forms the bonding interface between the acrylic and silicone elastomer. Mechanical preparations can also be made on the housing to increase the surface area for bonding. The shear bond strength of this interface should be greater than the attraction forces provided by magnets $(3.41-7.69 \mathrm{~N})^{33,34}$ or bars $(5-7 \mathrm{~N})^{35}$ to prevent any debonding.

\section{Maintenance and aftercare}

For long-term usage of the prosthesis, proper maintenance and aftercare are required. Lifelong commitment is one of the prerequisites for a patient undergoing rehabilitation of orbital defect with facial prosthesis. Following prosthesis delivery and usage, most commonly observed problems are margin tear, loss of retentive elements, and discoloration. ${ }^{32,37}$ The discoloration of silicone can be associated with daily wear, pollution, effect of cleansing agents, and long exposures to UV radiation that can also accelerate the degeneration. ${ }^{38}$ For implant-retained facial prosthesis, inflammation around the implant-abutment interface is one of the most commonly seen complications. Due to lack of proper seal between the abutment and the skin, any collection of debris and crusting can act as potential irritants and lead to the inflammation of this interface. ${ }^{39}$ The prosthesis should be designed to leave 1.5-2 $\mathrm{mm}$ space between the skin and the acrylic substructure ${ }^{40}$ to permit easy hygiene maintenance. Daily hygiene maintenance protocol should include cleaning the implant-abutment interface and the retentive components. For mild-to-moderate soft tissue reactions (Holgers' grade $2-3),{ }^{41}$ topical antimicrobial ointments are suggested, but for severe chronic reactions, the abutment may need to be removed to allow the tissues to heal.

\section{Future aspects}

In the field of medicine, the use of improved imaging techniques, such as $\mathrm{CT}$ and magnetic resonance imaging, has enabled significant improvements in how diseases are diagnosed, visualized, and treated. The digitalized images obtained from these techniques can be viewed from different angles and can be virtually reconstructed three dimensionally, which is not possible with the traditional methods of two-dimensional radiographs. In dentistry, these technologies have especially been useful in treatment planning for dental implants, where the anatomy of the osteotomy site can be visualized presurgically. Depending on the findings, the surgical protocols can also be adjusted such that the entire surgical procedure becomes more prosthetically driven. Virtual planning makes the treatment more accurate and predictable. Furthermore, fabrication of physical model also simplifies communication between the physicians and the patients.

Rapid prototyping uses the recorded digitalized data to reconstruct a physical model, by using either additive or subtractive method, with raw materials such as power, liquid, or solid..$^{42}$ Prototyping has been largely used in maxillofacial prosthetics to create accurate three-dimensional models as conventional impression techniques can cause deformation of soft tissues and introduce inaccuracies. The steps for rapid prototyping involve scanning of the patient's face using three-dimensional depth-sensing cameras and laser scanning 


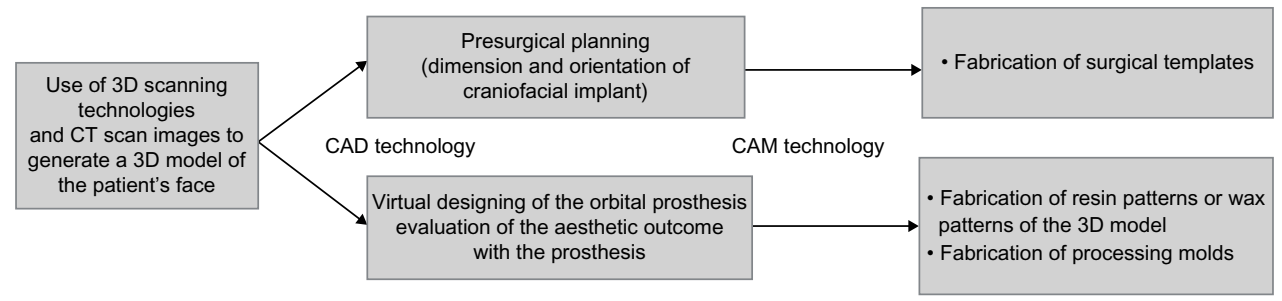

Figure 2 Application of computer aided design (CAD) and computer aided manufacturing (CAM) technologies in orbital prosthesis rehabilitation. Abbreviation: CT, computer tomography.

or phase measuring profilometry, and computer software is used to reconstruct the patient's face from the acquired data. ${ }^{43} \mathrm{Li}$ et al described a method to create a virtual model of the patient's face using CT scan images. The data were used to reconstruct an isosymmetrical model of the patient's normal orbit, which was then adapted on the defect side at the suitable position. ${ }^{44}$ As the traditional impression and intricate wax sculpturing procedures are eliminated, there is significant reduction in the overall prosthesis fabrication time. A corresponding STL (STereoLithography) file of the three-dimensional model is created, sliced into thin layers of $0.01 \mathrm{~mm}$ using software, and a physical model is produced in increments with resin or wax since a direct fabrication of a prototype with silicone elastomer is still not possible. Jiao et al used stereolithography to create a resin pattern of the patient's anatomy from a liquid polymer resin. However, the resin pattern needed to be duplicated in wax and tried on the patient for final fit and adaptation. ${ }^{45}$ A newer method of rapid prototyping, selective laser sintering, was introduced recently that permits direct fabrication of the three-dimensional model in wax patterns, using wax powder or polystyrene. Since the wax pattern is directly fabricated, inaccuracies produced with the conventional duplication steps are avoided. However, both forms of rapid prototyping cannot accurately reproduce skin texture details, follicular orifices, and thin adaptable margins. The wax pattern still needs to be adapted clinically on the patient and textured before the final prosthesis is fabricated. ${ }^{46}$ Nevertheless, the final prosthesis made with the help of rapid prototyping produces more aesthetic and refined results as compared to hand sculpturing.

The surgical placement of implants in the craniofacial bone is difficult due to the curvature and span of the orbital bone. Vital structures such as the air sinuses, cerebral tissues, and nerves are also present in the orbital area, which should be avoided during implant placement. Conventional methods of presurgical assessment of bone thickness at the osteotomy site using two-dimensional radiographs and twodimension section of the CT image can lead to inaccuracies. A higher surgical risk is also present as a majority of the decision making regarding the implant angulation, site, and size of the implant is based on intuition during the time of the surgery. This makes the entire surgical procedure technically demanding, requiring high-skilled and experienced surgeons. The use of computer-aided design along with stereolithography techniques can help in surgical planning and reducing overall time, cost, and precision required during implant placement. The three-dimensional data obtained from the CT can be used to virtually plan the exact site, depth, and angulation for implant placement, avoiding important vital structures. Li et $\mathrm{al}^{44}$ used quaternion interpolation techniques to compute the normal vector at various points along the curvature of the orbital bone and to visualize the potential implant sites along the orbital bone. Based on these predetermined locations, an accurate template can be milled and used during the actual surgery. ${ }^{47}$ Three-dimensional planning and the use of computer-aided design and manufacturing (CAD-CAM) have greatly improved the predictability of the treatment, such that the location, angulation, depth, and dimensions of the implants can be completely predetermined, and the final outlook of the prosthesis visualized before the treatment is initiated. The current applications of CAD-CAM technology in orbital prosthesis rehabilitation are summarized in Figure 2.

\section{Conclusion}

Prosthodontic rehabilitation remains a viable treatment option to surgical reconstruction, and the advent of modern silicone elastomers and application of osseointegration concept has helped to overcome many limitations of the conventional methods of retention. Due to lack of strong evidence-based studies, the protocol for implant placement in irradiated bone and the implication of HBO therapy has not been clearly stated nor followed. Incorporation of digital technology has helped to increase the accuracy and predictability of the treatment and to reduce the overall treatment time. With future advancements in material science and technology, the rehabilitation of orbital defects can be further improved and simplified. 


\section{Disclosure}

The authors report no conflicts of interest in this work.

\section{References}

1. Mueller S, Hohlweg-Majert B, Buergers R, et al. The functional and aesthetic reconstruction of midfacial and orbital defects by combining free flap transfer and craniofacial prosthesis. Clin Oral Investig. 2015;19(2):413-419.

2. Archontaki M, Stavrianos SD, Rapidis AD. Free microvascular tissue transfer for the reconstruction of midfacial defects in oncological patients. Med Oral Patol Oral Cir Bucal. 2010;15(5):746-751.

3. Li D, Jie Y, Liu H, Liu J, Zhu Z, Mao C. Reconstruction of anophthalmic orbits and contracted eye sockets with microvascular radial forearm free flaps. Ophthal Plast Reconstr Surg. 2008;24(2):94-97.

4. Motomura H, Iguchi H, Hatano T, et al. Dynamic eye socket reconstruction after extensive resection of midfacial malignancies: preliminary results using temporalis transfer. Acta Otolaryngol. 2014;134(11): 1205-1210.

5. Beumer J, Curtis TA, Marunik MT. Maxillofacial Rehabilitation: Prosthodontic and Surgical Consideration. St. Louis: Ishiyaku Euro America, Inc; 1996.

6. Federspil PA. Implant-retained craniofacial prostheses for facial defects. GMS Curr Top Otorhinolaryngol Head Neck Surg. 2009;8:1-16.

7. Craig RG, Powers JM. Restorative Dental Materials. Philadelphia: Mosby, Inc; 2002.

8. Montgomery PC, Kiat-Amnuay S. Survey of maxillofacial prostheses materials used. J Prosthodont. 2010;19(6):482-490.

9. Filié HM, Coelho GM, Micheline DSD, Moreno A, Filipe DN, Alves PA. Color stability of maxillofacial silicone with nanoparticle pigment and opacifier submitted to disinfection and artificial aging. J Biomed Opt. 2011;16(9):095004-1-095004-6.

10. Bellamy K, Limbert G, Waters MG, Middleton J. An elastomeric material for facial prostheses: aynthesis, experimental and numerical testing aspects. Biomaterials. 2003;24(27):5061-5066.

11. Sanchez RA, Moore DJ, Cruz DL, Chappell R. Comparison of the physical properties of two types of polydimethyl siloxane for fabrication of facial prostheses. J Prosthet Dent. 1992;67(5):679-682.

12. Udagama A. Urethane-lined silicone facial prostheses. J Prosthet Dent. 1987;58(3):351-354.

13. Chu CC, Fischer TE. Evaluation of sunlight stability of polyurethane elastomers for maxillofacial use: II. J Biomed Mater Res. 1979;13(6):965-974.

14. Tran NH, Scarbecz M, Gary JJ. In vitro evaluation of color change in maxillofacial elastomer through the use of an ultraviolet light absorber and a hindered amine light stabilizer. J Prosthet Dent. 2004;91(5):483-490.

15. Han Y, Powers JM, Kiat-Amnuay S. Effect of opacifiers and UV absorbers on pigmented maxillofacial silicone elastomer, part 1: color stability after artificial aging. J Prosthet Dent. 2013;109(6):397-401.

16. Lemon JC, Chambers MS, Jacobsen ML, Powers JM. Color stability of facial prostheses. J Prosthet Dent. 1995;74(6):613-618.

17. Bryant AW, Schaaf NG, Casey DM. The use of a photoprotective agent to increase the color stability of a tinted extraoral prosthetic silicone. J Prosthodont. 1994;3(2):96-102.

18. Han Y, Kiat-amnuay S, Powers JM, Zhao Y. Effect of nano-oxide concentration on the mechanical properties of a maxillofacial silicone elastomer. J Prosthet Dent. 2008;100(6):465-473.

19. Veerareddy C, Nair KC, Reddy GR. Simplified technique for orbital prosthesis fabrication: a clinical report. J Prosthodont. 2012;21(7):561-568.

20. Hu X, Pan X, Johnston WM. Effects of pigments on dynamic mechanical properties of a maxillofacial prosthetic elastomer. J Prosthet Dent. 2014;112(5):1298-1303.

21. Granström G. Craniofacial osseointegration. Oral Dis. 2007;13(3): 261-269.
22. Chang TL, Garrett N, Roumanas E, Beumer J 3rd. Treatment satisfaction with facial prostheses. J Prosthet Dent. 2005;94(3):275-280.

23. Curi MM, Oliveira MF, Molina G, et al. Implants in the rehabilitation of craniofacial defects: Implant and prosthesis survival rates peri-implant soft tissue evaluation. J Oral Maxillofac Surg. 2012;70(7):1551-1557.

24. Granström G, Bergström K, Tjellström A, Brånemark PI. A detailed analysis of titanium implants lost in irradiated tissues. Int J Oral Maxillofac Implants. 1994;9(6):653-662.

25. Nishimura RD, Roumanas E, Moy PK, Sugai T, Freymiller EG. Osseointegrated implants and orbital defects: U.C.L.A. experience. J Prosthet Dent. 1998;79(3):304-309.

26. Granström G. Placement of dental implants in irradiated bone: the case for using hyperbaric oxygen. J Oral Maxillofac Surg. 2006;64(5):812-818.

27. Moran WJ, Toljanic JA, Panje WR. Implant-retained prosthetic rehabilitation of orbital defects. Arch Otolaryngol Head Neck Surg. 1996;122(1):46-50.

28. Toljanic JA, Eckert SE, Roumanas E, et al. Osseointegrated craniofacial implants in the rehabilitation of orbital defects: an update of a retrospective experience in the United States. J Prosthet Dent. 2005;94(2):177-182.

29. Wolfaardt JF, Wilkes GH, Parel SM, Tjellström A. Craniofacial osseointegration: the Canadian experience. Int J Oral Maxillofac Implants. 1993;8(2):197-204.

30. Roumanas ED, Chang TL, Beumer J. Use of osseointegrated implants in the restoration of head and neck defects. $J$ Calif Dent Assoc. 2006;34(9):711-718.

31. Wolfaardt J, Gehl G, Farmand M, Wilkes G. Indications and methods of care for aspects of extraoral osseointegration. Int J Oral Maxillofac Surg. 2003;32(2):124-131.

32. Tjellström A, Granström G. One-stage procedure to establish osseointegration: a zero to five years follow-up report. J Laryngol Otol. 1995;109(7):593-598

33. Al-Salehi SK, Calder ID, Lamb DJ. Magnetic retention for obturators. J Prosthodont. 2007;16(3):214-218.

34. Voigt A, Christ S, Klein M. Experimental analysis of retention forces of different magnetic devices for bone-anchored auricular facial prostheses. Int J Oral Maxillofac Surg. 2008;37(7):664-668.

35. Lehmann KM, Arnim FV. Studies on the retention forces of snap-on attachments. Quintess Dent Technol. 1978;7:45-48.

36. Faulkner G, Wolfaardt J, del Valle V. Console abutment loading in craniofacial osseointegration. Int J Oral Maxillofac Implants. 1998;13:245-252.

37. Ariani N, Visser A, van Oort RP, et al. Current state of craniofacial prosthetic rehabilitation. Int J Prosthodont. 2013;26(1):57-67.

38. Kulkarni RS, Nagda SJ. Colour stability of maxillofacial silicone elastomers: a review of the literature. Eur J Prosthodont Restor Dent. 2014;22(3):108-115.

39. Holgers KM, Thomsen P, Tjellstrom A, Bjursten LM. Immunohistochemical study of the soft tissue around long-term skin-penetrating titanium implants. Biomaterials. 1995;16(8):611-616.

40. Karakoca S, Aydin C, Yilmaz H, Bal BT. Survival rates and peri-implant soft tissue evaluation of extraoral implants over a mean follow-up period of three years. J Prosthet Dent. 2008;100(6):458-464.

41. Holgers KM, Tjellstrom A, Bjursten LM, Erlandsson BE. Soft tissue reactions around percutaneous implants: a clinical study on skin-penetrating titanium implants used for bone-anchored auricular prostheses. Int J Oral Maxillofac Implants. 1987;2(1):35-39.

42. Goiato MC, Santos MR, Pesqueira AA, Moreno A, dos Santos DM, Haddad MF. Prototyping for surgical and prosthetic treatment. J Craniofac Surg. 2011;22(3):914-917.

43. Davis BK. The role of technology in facial prosthetics. Curr Opin Otolaryngol Head Neck Surg. 2010;18(4):332-340.

44. Li S, Xiao C, Duan L, Fang C, Huang Y, Wang L. CT image-based computer-aided system for orbital prosthesis rehabilitation. Med Biol Eng Comput. 2015;53(10):943-950. 
45. Jiao T, Zhang F, Huang X, Wang C. Design and fabrication of auricular prostheses by CAD/CAM system. Int J Prosthodont. 2004;17(4):460-463.

46. Feng Z, Dong Y, Zhao Y, et al. Computer-assisted technique for the design and manufacture of realistic facial prostheses. Br J Oral Maxillofac Surg. 2010;48(2):105-109.
47. Goh BT, Teoh KH. Orbital implant placement using a computer-aided design and manufacturing (CAD/CAM) stereolithographic surgical template protocol. Int J Oral Maxillofac Surg. 2015;44(5):642-648.
Open Access Surgery

\section{Publish your work in this journal}

Open Access Surgery is an international, peer-reviewed, open access journal that focuses on all aspects of surgical procedures and interventions. Patient care around the peri-operative period and patient outcomes post surgery are key topics. All grades of surgery from minor cosmetic interventions to major surgical procedures are covered. Novel techniques

Submit your manuscript here: http://www.dovepress.com/open-access-surgery-journal

\section{Dovepress}

and the utilization of new instruments and materials, including implants and prostheses that optimize outcomes constitute major areas of interest. The manuscript management system is completely online and includes a very quick and fair peer-review system. Visit http://www.dovepress.com/ testimonials.php to read real quotes from published authors. 